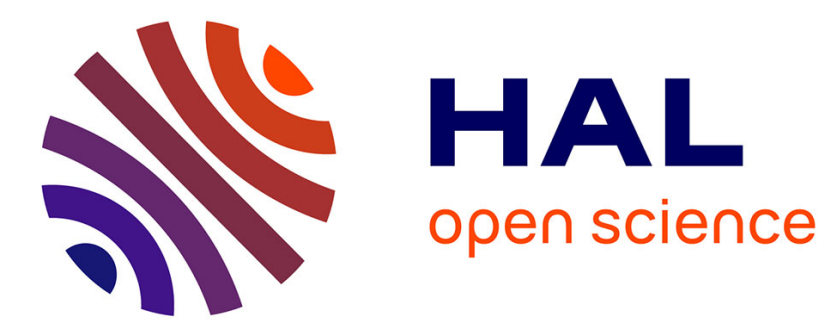

\title{
Situations de travail et mobilisations ouvrières en Méditerranée
}

\author{
Amin Allal, Michele Scala, Élisabeth Longuenesse
}

\section{To cite this version:}

Amin Allal, Michele Scala, Élisabeth Longuenesse. Situations de travail et mobilisations ouvrières en Méditerranée. Confluences Méditerranée , 2019, 111, pp.9-14. 10.3917/come.111.0009 . halshs03527156

\section{HAL Id: halshs-03527156 \\ https://shs.hal.science/halshs-03527156}

Submitted on 15 Jan 2022

HAL is a multi-disciplinary open access archive for the deposit and dissemination of scientific research documents, whether they are published or not. The documents may come from teaching and research institutions in France or abroad, or from public or private research centers.
L'archive ouverte pluridisciplinaire HAL, est destinée au dépôt et à la diffusion de documents scientifiques de niveau recherche, publiés ou non, émanant des établissements d'enseignement et de recherche français ou étrangers, des laboratoires publics ou privés. 
Situations de travail et mobilisations ouvrières en Méditerranée Amin Allal, Michele Scala, Élisabeth Longuenesse

\author{
Dans Confluences Méditerranée 2019/4 ( $\left.\mathbf{N}^{\circ} \mathbf{1 1 1}\right)$, pages 9 à 14
}

Du Maroc à la Turquie, en passant par l'Algérie, la Tunisie, l'Égypte, la Grèce, l'Italie, le Liban ou la Jordanie [1][1]Allal A. (2010), «Réformes néolibérales, clientélisme et..., le renouvellement des formes de protestation, plus ou moins directement liées au travail, est désormais un constat transméditerranéen. Dans ces situations contrastées on retrouve le même processus d'affaiblissement des syndicats et de fragilisation du rapport salarial et des protections sociales qui l'accompagnaient. D'un côté, la part de la grande industrie s'est réduite au profit du secteur des services, y compris des services à la personne, de l'autre, l'individualisation des tâches, le développement de la sous-traitance et les délocalisations, ont permis de contourner les contraintes associées à la norme salariale. La financiarisation de l'économie mondiale a ainsi entrainé un double mouvement de marchandisation accélérée des rapports humains et de pression accrue sur une force de travail cantonnée de façon croissante à des fonctions de service (transport et distribution de biens de consommation, activités intellectuelles et culturelles, volontariat social et caritatif, services de toute sorte).

2Ces évolutions prennent des formes différentes selon les contextes. Le poids du travail migrant et des circulations pendulaires des travailleurs (dans l'agriculture, dans la construction, dans les services), l'exploitation des femmes et des enfants, sont plus ou moins marqués d'un pays à l'autre. L'absence de régulation par le droit, le poids des relations de clientèle semblent plus fréquents au Sud. Mais d'un bout à l'autre de la Méditerranée, on s'aperçoit vite que ces dynamiques traversent les pays et les secteurs d'activité : une fragmentation et une précarisation du travail qui prennent de nouvelles formes et touchent de nouvelles catégories sociales, incluant de plus en plus largement les nouvelles générations de jeunes diplômés, y compris dans les secteurs réputés les plus modernes de production de biens et de services.

3 Souvent présentés comme une tendance plus générale à la déstabilisation du salariat, les processus dits d'informalisation (au Sud) et de précarisation (au Nord) méritent d'être davantage interrogés. D'une part, l'informel n'est pas cette catégorie résiduelle, minoritaire ou même exceptionnelle par rapport à la norme du travail « formel » (i.e. le travail salarié encadré et protégé par la Loi) [2][2]Le dernier rapport de la Banque Mondiale montre que.... D'autre part, si le débat autour de la précarité s'impose en Europe à partir des années 1970, ce n'est pas parce qu'elle est en soi nouvelle, mais plutôt parce qu'elle s'institutionnalise dans le cadre de la loi, en devenant « visible » et donc « dicible » [3][3]Anne-Sophie Beau, Un siècle d'emplois précaires. Patron-ne-s et.... En outre, comme le montrent bien les enquêtes de terrain réunies dans ce dossier, une attention exclusivement portée aux statuts de l'emploi et aux protections afférentes ne rend que partiellement compte des mutations contemporaines du travail. Les clivages de classe, de race, de nationalité et de genre participent aussi à la fragmentation des statuts et des canaux d'accès aux protections via le travail.

$\underline{4} \hat{\mathrm{A}}$ ces mutations du travail correspond une transformation des formes de résistance et de contestation [4][4]Allal A., Catusse M., Emperador Badimon M. (2018), Quand.... Inféodées aux pouvoirs en place, violemment réprimées par les régimes autoritaires, ou prisonnières de logiques bureaucratiques ou clientélistes, les grandes organisations syndicales se voient de plus en plus souvent contournées par des mobilisations issues de la base. On voit ainsi apparaître des 
mouvements plus ponctuels ou sectoriels, parfois violents ou désespérés, mais aussi de nouvelles formes de coopération ou d'entraide pour s'organiser ou faire face à des situations de crise [5][5]Peyman E. (2015), Against all odds, labor activism in the....

5 La dizaine d'articles ici rassemblés abordent ces différentes questions à partir d'études de cas, au niveau local (l'entreprise Technoverre à Tunis, les ouvriers des chantiers navals au Pirée, les ouvriers agricoles dans l'Agro Pontino en Italie), sectoriel (les enseignants marocains, les ouvriers de l'industrie textile en Jordanie, les ouvriers agricoles migrants en Italie, les travailleurs des ONG caritatives syriennes au Liban), ou national (en Turquie et en Égypte, où l'état d'urgence et le durcissement des régimes se font durement sentir sur les mobilisations ouvrières, en Algérie et au Liban, où l'on observe au contraire une floraison de mobilisations en dehors des structures officielles).

6Le croisement de ces cas d'études nous montre comment les effets de la mondialisation des marchés et des politiques néolibérales se traduisent par la fermeture et la délocalisation d'entreprises, dans une logique de profit de court terme. Ainsi, à Tunis, une entreprise dont les ouvriers avaient pourtant développé une expertise remarquable, est rachetée par un petit investisseur qui remplace les ateliers de production par des entrepôts de marchandises dans un projet d'import-export plus «rentable» (Mohamed Slim Ben Youssef); tandis que des investisseurs asiatiques délocalisent une partie de leur production textile vers la Jordanie, pour accéder au marché américain (Taher Labadi). La mondialisation des marchés, c'est aussi le recours à une main-d'œuvre étrangère, dont la mobilité est à la fois entravée et planifiée, et de ce fait mobilisable dans des conditions d'asservissement et de précarité accrue : ouvriers agricoles indiens en Italie (Marco Omizzolo et al.), ouvriers du textile asiatiques en Jordanie, demandeurs d'asile africains employés « bénévolement » dans l'entretien des espaces publics en Italie (Simone Di Cecco). La croissance du secteur de l'humanitaire dans les régions en crise est une autre forme de cette mondialisation, qui voit se côtoyer ONG locales et internationales, employant expatriés, salariés et bénévoles de diverses nationalités, entraînant hiérarchisation des statuts et précarisation des locaux : le cas des employés syriens d'ONG au Liban apparaît emblématique, dans un contexte où Syriens et Palestiniens sont exclus de droit de tout emploi qualifié (Leo Fourn).

7Le recul du salariat protégé et son corollaire, la précarisation du travail, sont une dimension massive de l'évolution des marchés du travail qui ne concerne pas que les travailleurs migrants. Si le travail non déclaré, l'emploi à la tâche, dépendant de la demande ou des commandes des donneurs d'ordre, est une caractéristique ancienne de certains marchés où les toutes petites entreprises dominent, comme le montre le cas des chantiers navals du Pirée (Manos Spyridakis), il (re) devient une stratégie systématique, non seulement des grands acteurs économiques privés, mais aussi des employeurs étatiques, sous la pression des bailleurs et institutions internationales. Au Maroc (Abdellatif Zeroual et Abdellah Lefnatsa), comme en Algérie (Siham Beddoubia), les fonctionnaires titulaires dans l'éducation et la santé sont de plus en plus souvent remplacés par des contractuels précaires, qui se mobilisent aux côtés des diplômés chômeurs. En Italie, les communes compensent la réduction de leurs budgets par l'emploi de chômeurs en fin de droit et de «bénévoles » demandeurs d'asile. La popularité croissante de la notion de « volontariat» dans le champ de l'humanitaire et de l'aide au développement masque mal le recours à une main d'œuvre qualifiée, sous payée et non protégée.

8Dans les cas de figures interrogés dans ce dossier, la dégradation des conditions de travail et d'emploi s'accompagne souvent de conditions de vie de plus en plus dures. En Grèce, les anciens ouvriers qualifiés des chantiers navals se voient non seulement contraints d'accepter 
des emplois ne correspondant plus à leur niveau de compétence, mais aussi, pour survivre, de recourir à l'aide des réseaux familiaux et villageois. Les ouvriers étrangers des QIZ en Jordanie sont quant à eux entassés dans des camps, à l'écart des agglomérations et de la société locale, et se voient déduire de leur rémunération le «coût» de leur hébergement de façon discrétionnaire. Le cas des ouvriers sikhs de l'Agro Pontino révèle aussi que les arrangements habituellement accolés à l'informel comme ceux issus de l'intermédiation illicite de maind'œuvre (caporalato), s'appuient sur des dispositifs tout à fait formalisés de recrutement, ce qui montre, s'il en était besoin, qu'il n'est pas aisé de lire ces pratiques sans considérer leur étroite connexion.

9En lien avec ces mutations du travail, les contributions à ce dossier évoquent toutes, au cœur de leur propos ou de façon corollaire, la diversité des formes de résistance et de luttes, les reconfigurations syndicales, la relation entre luttes sociales et luttes démocratiques.

$\underline{10 U n}$ peu partout, à des degrés divers, apparaît une défiance croissante à l'égard d'« organisations syndicales "traditionnelles" en crise et affaiblies à la fois par leur évolution interne (la bureaucratisation), les tactiques du pouvoir (répression, cooptation) et les transformations du monde du travail »(Lefnatsa et Zeroual), comme au Maroc ou au Liban (Hariri et Scala) ; mais aussi gangrenées par la corruption (Spyridakis). Cette défiance suscite l'émergence d'organisations issues de la base, à la suite de mobilisations concrètes. C'est ainsi le cas des enseignants contractuels au Maroc mais aussi, dès les années 1990, et, surtout après 2000, de nombreuses catégories d'employés de l'État en Algérie (Beddoubia). À la même époque, naissent les premiers syndicats indépendants en Égypte, tandis que de grandes grèves ouvrières en dehors de toute organisation, soutenues par un mouvement de jeunes, annoncent les prodromes de la révolution de 2011 (Françoise Clément). En Égypte, mais aussi en Turquie (Isil Erdinç), la répression syndicale, corollaire de l'état d'urgence, freine, mais n'empêche pas, la poursuite de mobilisations, qui s'organisent désormais souvent au niveau des entreprises.

$\underline{11 A u-d e l a ̀ ~ d e s ~ l u t t e s ~ p o u r ~ l ' e m p l o i, ~ l e s ~ s a l a i r e s, ~ l e s ~ c o n d i t i o n s ~ d e ~ v i e ~ e t ~ d e ~ t r a v a i l, ~ u n e ~ a u t r e ~}$ bataille se livre, à laquelle on n'a jusqu'à présent que peu prêté attention, autour du sens du travail, de la dignité du travailleur, de la figure du «bon travailleur ». Tandis que les ouvriers de Technoverre à Tunis se battent non seulement pour l'emploi, mais aussi pour la reconnaissance de leur savoir, et pour la défense de leur outil de travail, pour les Grecs des chantiers de Perama, si le travail s'arrête, le cœur de Perama cesse de battre et le quartier meurt ( The area is to the workers what the heart is to man. If it stops ticking, he dies »). Dans un registre différent, mais qui leur fait écho, les travailleurs bénévoles syriens des ONG humanitaires au Liban sont prêts à travailler sans protection et sous rémunérés, parce que ce travail est pour eux indissociable d'un engagement auprès de leurs concitoyens. À l'inverse, la figure du «bon travailleur», promu par les employeurs des QIZ en Jordanie ou ceux des entreprises agricoles du Latium, ou du « bon migrant » accueilli dans les centres d'hébergement qui accepte de travailler gratuitement, légitime des formes de surexploitation de leur travail : travail gratuit pour les uns, réduction du salaire sous prétexte de déduction du coût du logement pour d'autres, allongement de la journée de travail pour la plupart. Mais en Italie comme en Jordanie et ailleurs, loin d'être toujours dupes, ces travailleurs réussissent parfois à surmonter les facteurs de division qu'entraine la multiplicité des origines et des langues et à organiser la résistance ou la protestation.

12Finalement, il apparaît que les formes supposées « atypiques » du travail ne peuvent plus être appréhendées comme des effets pervers et dérogatoires du développement capitaliste, car au «Sud » comme au « Nord » de la Méditerranée, et bien au-delà, l’hétérogénéité du travail, de 
ses modes d'organisation, de soumission et de protection s'impose. Les mutations mises en lumière dans ce dossier suggèrent ainsi de réinterroger nos catégories d'analyse : que signifie le travail aujourd'hui ? Quelle relation entre travail, emploi, volontariat, bénévolat ? Peut-on se contenter de la dichotomie travail formel-informel ? Précarité-stabilité ? Quel statut pour le travail et le travailleur dans la société ? En quels termes parler d'exploitation du travail ? Comment s'articulent exploitation, domination, oppression ? Enfin, comment penser la relation entre actions syndicales et mobilisations sociales plus larges liées au travail, dans un contexte où luttes locales et luttes globales paraissent de plus en plus en résonance.

\section{Notes}

- [1]

Allal A. (2010), «Réformes néolibérales, clientélisme et protestation en situation autoritaire. Les mouvements contestataires dans la région de Gafsa en Tunisie (2008) », Politique africaine, $\mathrm{n}^{\circ}$ 117, pp. 107-126. Catusse M., Vairel F. (2010), «Question sociale et développement : les territoires de l'action publique et de la contestation au Maroc », Vol. 4, n. 120, p. 5-23. Beinin J., Duboc M. (2014), « The Egyptian workers movement before and after the 2011 popular uprising, Socialist register », Merlin Press, London. Kretsos L., Vogiatzoglou M. (2015). Lost in the Ocean of Deregulation ? : The Greek Labour Movement in a Time of Crisis. Relations Industrielles/Industrial Relations, 70 (2). Perrotta D.,Sacchetto D. (2014), "Migrant Farmworkers in Southern Italy: Ghettoes, Caporalato and Collective Action", in Workers of the World. International Journal on Strikes and Social Conflicts, Vol. I, n. 5. Scala M. (2015), "Clientélisme et contestation. L'exemple de la mobilisation des travailleurs de Spinneys (Liban)», Confluences méditerranée, $\mathrm{n}^{\circ}$ 92, 113-123. Fioroni C. (2015), «From the Everyday to Contentious Collective Actions : The Protests of the Employees of the Jordan Phosphate Mines Company in $2011 »$, Workers of the World. International Journal on Strikes and Social Conflict, Vol. 7, n. 1.

- [2]

Le dernier rapport de la Banque Mondiale montre que l'« informel » concerne $75 \%$ des emplois dans les pays dits «en développement: BM, «Le travail en mutation», Washington DC, 2019, p. 7.

- $[3]$

Anne-Sophie Beau, Un siècle d'emplois précaires. Patron-ne-s et salarié-e-s dans le grand commerce $\left(X I X^{e}-X X^{e}\right)$, Paris, Payot, 2004.

- $\quad 4]$

Allal A., Catusse M., Emperador Badimon M. (2018), Quand l'industrie proteste. Fondements moraux des (in) soumissions ouvrières, Presses Universitaires de Rennes, Rennes.

- $\quad[5]$ 
Peyman E. (2015), Against all odds, labor activism in the Middle East and North Africa, Workers of the World. International Journal on Strikes and Social Conflicts, vol. 7/1. Quijoux M. (2017), «Les conflits du travail dans le monde aujourd'hui », Critique internationale, Vol. $1, \mathrm{n}^{\circ} 74$ 